\title{
Uso de las prácticas de comercialización de las PYME de la Ciudad de México y su relación con la competitividad
}

\section{Use of SME marketing practices in Mexico City and their relationship to competitiveness}

\begin{abstract}
Dra. María Luisa Saavedra-García es profesora titular de tiempo completo de la Universidad Nacional Autónoma de México (maluisasaavedra@yahoo.com) (https://orcid.org/0000-0002-3297-1157)
\end{abstract}

Dra. María del Rosario Demuner-Flores es profesora investigadora de tiempo completo de la Universidad Autónoma del Estado de México (demuner7@yahoo.com) (https://orcid.org/0000-0002-4542-9113)

Dra. Elsa Esther Choy-Zevallos es docente investigadora de la Universidad Nacional Mayor de San Marcos, Perú (echoyz@unmsm.edu.pe) (https://orcid.org/0000-0001-8580-8495)

\section{Resumen}

El objetivo de esta investigación consiste en conocer las prácticas de comercialización que utilizan las PYME de la Ciudad de México y determinar si existe relación entre estas y la competitividad interna, considerando el tamaño y el sector en que se desempeñan. El método de investigación es empírico con un alcance descriptivo y correlacional, se trabajó con una muestra de 400 PYME recolectando datos a través de un trabajo de campo, se aplicó el mapa de competitividad del BID adaptado por Saavedra (2014b), que mide la competitividad interna de las empresas considerando 8 áreas, para este trabajo sólo se analizó el área de comercialización que incluye las prácticas: Plan de mercado, estrategias comerciales, conocimiento de competidores, determinación de precios, productos nuevos, marketing, servicio al cliente, fuerza de ventas y sistemas de distribución. Los resultados muestran un bajo nivel de aplicación de las prácticas de Comercialización, determinado por el uso de prácticas básicas y una relación significativa entre las prácticas de comercialización y la competitividad, lo cual implica la importancia del uso de prácticas de comercialización para impulsar la competitividad de las PYME.

\begin{abstract}
The aim of this research is to find out about the marketing practices used by Smes in Mexico City and to determine whether they are related to internal competitiveness, considering the size and sector in which they operate. The research method is empirical with a descriptive and correlational scope, working with a sample of 400 Smes collecting data through a field study, applied the IDB competitiveness map adapted by Saavedra (2014b), which measures the internal competitiveness of the companies considering 8 areas, for this work was only analyzed the area of marketing that includes the practices: Market plan, commercial strategies, knowledge of competitors, pricing, new products, marketing, customer service, sales force and distribution systems. The results show a low level of application of marketing practices, determined by the use of basic practices and a significant relationship between marketing practices and competitiveness, which implies the importance of the use of marketing practices to boost the competitiveness of Smes.
\end{abstract}

\section{Palabras clave I keywords}

Competitividad, PYME, marketing, rentabilidad, competidores, clientes, ventas, productos. Competitiveness, SMEs, marketing, profitability, competitors, customers, sales, products.

Cómo citar: Saavedra-García, M.L., Demuner-Flores, M. del R., y Choy- Zevallos, E. E. (2020). Uso de las prácticas de comercialización de las PYME de la Ciudad de México y su relación con la competitividad. Retos Revista de Ciencias de la Administración y Economía, 10(20), pp. 283-305. https://doi.org/10.17163/ret.n20.2020.06 


\section{Introducción}

Las pequeñas y medianas empresas $\left(\mathrm{PYME}^{1}\right)$ son indispensables en el desarrollo social de las naciones, un aspecto destacable es su alto índice de empleabilidad (Saavedra, 2014a), según datos oficiales publicados por INEGI (2020) el 95\% del total de las unidades económicas en México son microempresas, $4 \%$ son pequeñas y $0.8 \%$ medianas, mismas que aportan el 52\% del Producto Interno Bruto (PIB) y generan el $68.4 \%$ del empleo. En cuanto a la clasificación sectorial el $12.19 \%$ pertenecen al sector industria, el $46.65 \%$ son empresas del sector comercio, el 39.15\% corresponde a empresas del sector de servicios no financieros y el $2.01 \%$ al resto de actividades económicas (INEGI, 2020), lo cual permite ver que en las PYME mexicanas predominan los sectores comercio y servicios.

Así también, en México de acuerdo con INEGI (2019) las PYME tiene muchas carencias sobre todo en el aspecto administrativo, pues solo el 15\% capacitan a sus empleados, únicamente el 14\% realizan acciones e implementan un proceso de mejora continua para solucionar los problemas de producción, el 65\% no monitorean indicadores de desempeño, sólo un 3\% cuentan con un sistema contable que les permita registrar sus operaciones y obtener información financiera para la toma de decisiones.

Aunado a lo anterior, estudios antecedentes muestran que muchas PYME no adoptan prácticas de comercialización, o utilizan prácticas básicas, sin llegar a implementar estrategias de largo plazo y menos aún estrategias de mercadotecnia digital que les permitan alcanzar competitividad y permanencia en el largo plazo (SorinaDiana et al., 2013; Bretcu, 2014; Gutiérrez \& Nava, 2016). En el caso mexicano los pocos estudios existentes (Saavedra \& Saavedra, 2014; Alcántara \& Goytortúa, 2013; Peterson \& Crittenden, 2020) dan cuenta de la incipiente aplicación de las prácticas de mercadotecnia en las empresas de este sector. Así pues, aunque tienen conocimiento de estas prácticas, afrontan serias limitaciones para su implementación debido a su baja capacidad financiera, falta de conocimientos específicos sobre cómo implementar estas prácticas y falta de personal capacitado. Esta situación le estaría restando competitividad y la pone en desventaja ante un entorno interno y externo altamente competitivo; por otra parte, son escasos los estudios acerca de las prácticas de comercialización en las PYME, lo que genera desconocimiento acerca de las prácticas que utilizan las PYME y su impacto en la mejora del desempeño de estas empresas; los estudios antecedentes se han basado en las prácticas empresariales en general, sin especificar prácticas adecuadas para las PYME (Bocconcelli et al., 2018), de ahí la importancia de realizar esta investigación que tiene como objetivo, conocer las prácticas de comercialización que utilizan las PYME de la Ciudad de México. y comprobar si existe relación entre estas y la competitividad interna, medida con el mapa de competitividad del BID, considerando el tamaño y el sector en que se desempeñan. Es

1 Micro, Pequeñas y Medianas Empresas, en el caso de México: Se clasifican como micro las empresas que cuentan con hasta 10 trabajadores, con ingresos por ventas anuales de hasta 4 millones de pesos. Pequeñas las que cuentan con entre 11 y 30 trabajadores (sector comercio) y entre 11 y 50 trabajadores (sector Industria y Servicios), con ingresos por ventas anuales de entre 4.01 hasta 100 millones de pesos. Son Medianas las que cuentan con entre 51 a 250 trabajadores (sector industria), entre 51 a 100 trabajadores (Sector servicios), y entre 31 a 100 trabajadores (sector Comercio), que reporten ingresos por ventas anuales de entre 100.01 hasta 250 millones de pesos (Secretaría de Economía, 2009). 
oportuno aquí, hacer notar que este artículo corresponde a la tercera parte de un proyecto más amplio denominado "La determinación de la competitividad de las PYME en el Distrito Federal” que incluye ocho áreas de las empresas, utilizando el mapa de competitividad del BID (adaptado por Saavedra, 2014b) mismo que se describe en el apartado 2.1.

Este trabajo se encuentra dividido en tres apartados 1. Revisión de la literatura, donde se desarrollan las prácticas de comercialización en las PYME, la competitividad en las PYME y las prácticas de comercialización y su relación con la competitividad; 2. Método, en este apartado se describe el mapa de competitividad del BID y la operacionalización de las variables del estudio; 3. La competitividad de las PYME en la ciudad de México, aquí se presenta el desarrollo de la investigación que incluye los hallazgos preliminares y los hallazgos acerca de las prácticas de comercialización; por último, se presentan las conclusiones de este trabajo.

\section{Revisión de la literatura}

\subsection{Las prácticas de comercialización en las PYME}

Los estudios realizados acerca de las prácticas de comercialización destacan la utilidad de estas para lograr mejores rendimientos, la forma en que pueden ser implementadas, así como las prácticas más usadas por las PYME. En este contexto, Sheetal et al. (2012), refieren que el marketing como estrategia se basa en las 4P y es capaz de influir en el rendimiento de las pequeñas empresas, sin embargo, en la turbulencia actual del mercado se han desarrollado conceptos nuevos y emergentes tales como: mercadeo en red, mercadeo innovador, estandarización vs. adaptación y agrupamiento; factores de contingencia que se relacionan con: cuestiones de empresa, gestión, producto, mercado y cliente. Por otra parte, el marketing a través de tres dimensiones: Cultural, estratégico y táctico puede aplicarse en el caso de las PYME. Así también, Sheetal et al. (2012) afirman que solo un marketing efectivo podría ayudar a las PYME a ganar ventaja competitiva a través de un rendimiento superior utilizando estrategias de marketing competitivo, marketing mix y procesos de inteligencia de mercados, lo cual puede beneficiar a las pequeñas empresas en gran medida al tiempo que recomiendan la creación de redes de negocios. Por otra parte, Cepeda et al. (2017) y Petkovska et al. (2018) señalan que las PYME, gracias a su flexibilidad para adaptarse a las condiciones del mercado tiene la oportunidad de introducir nuevos y mejores productos, así como, aplicar soluciones de marketing de acuerdo a las oportunidades que van encontrando en el mercado.

En este mismo sentido Mehran y Zeinab (2020), refieren que el marketing convencional no es suficiente cuando las PYME enfrentan un ambiente turbulento de alta competencia, por lo que se hace necesario que adopten un marketing innovador buscando constantemente nuevas oportunidades en el mercado, con procesos, servicios y productos nuevos que logren colocar a la empresa en ventaja a la empresa frente a sus competidores.

Sin embargo, un estudio empírico realizado por Sheetal et al. (2012), reveló que las PYME no aplican estrategias de marketing adecuadas a su contexto y son débiles en la estrategia de diferenciación; sin embargo, siguen estrategias de precios de bajo 
costo, por lo que deben procurar la diferenciación en el desarrollo de productos, es decir buscar nichos de mercado con productos especializados. Así también, los pequeños fabricantes necesitan usar estrategias de posicionamiento selectivo del producto, diferentes para cada producto; por otra parte, necesitan mejorar sus canales de distribución con el fin de alcanzar más clientes y más mercados, así como, incorporar tecnología de e-marketing lo cual puede ayudar al pequeño empresario a alcanzar a un gran número de compradores (Sheetal et al. 2012). Asimismo, Saavedra y Saavedra (2014), encontraron que las PYME del estado de Hidalgo en México usan técnicas de mercadotecnia básicas como la fijación de precios con base en los costos de producción, en cuanto a la innovación menos de la tercera parte de los empresarios realiza innovación en los productos, concluyendo que tienen poca oportunidad de llegar a los mercados internacionales limitando así su competitividad.

Por su parte, Petkovska et al. (2018), en una muestra de 32 PYME de Macedonia encontraron que cerca de la mitad realizan estudios de mercado, la otra mitad manifestaron que no lo realizan por falta de recursos financieros, no tienen conocimientos suficientes y no cuentan con el personal capacitado, lo cual es coincidente con los hallazgos de Cohen (2017) que dan cuenta del escaso conocimiento de marketing que muestran los colaboradores de las PYME, donde solo la cuarta parte realizan actividades de promoción, 16\% usan encuestas de satisfacción a clientes y sólo el 12\% realizan actividades de mercadeo.

Con el fin de conocer las prácticas de comercialización que utilizan las PYME en la tabla 1, se presenta una revisión de los estudios antecedentes que han logrado identificar estas prácticas y la forma como son implementadas en las PYME.

\section{Tabla 1. Prácticas de Comercialización en las PYME}

\begin{tabular}{|l|l|l|}
\hline \multicolumn{1}{|c|}{$\begin{array}{c}\text { Prácticas de } \\
\text { comercialización } \\
\text { en las PYME }\end{array}$} & \multicolumn{1}{|c|}{ Autores } & \multicolumn{1}{|c|}{ Hallazgos } \\
\hline $\begin{array}{l}\text { Investigación de } \\
\text { mercados }\end{array}$ & $\begin{array}{l}\text { Zapata (2001), Rojas y Briceño } \\
\text { (2006), Coy et al. (2007), Alcántara } \\
\text { et al. (2013), Wai-Sum (2005), Do- } \\
\text { mínguez et al. (2012), Saavedra y } \\
\text { Saavedra (2014), Petkovska et al. } \\
\text { (2018) }\end{array}$ & $\begin{array}{l}\text { Es costoso de realizar y lo utilizan } \\
\text { tamaño del mercado y aumentar } \\
\text { sus ventas. Orientado al cliente. } \\
\text { Utilizan el método de observación } \\
\text { y entrevistas informales, con fuen- } \\
\text { tes secundarias de información. }\end{array}$ \\
\hline $\begin{array}{l}\text { Mercadotecnia orienta- } \\
\text { da a la producción }\end{array}$ & $\begin{array}{l}\text { Rojas y Briceño (2006), Domínguez } \\
\text { et al. (2012), Monferrer (2013) }\end{array}$ & $\begin{array}{l}\text { Fabricar al mínimo costo produc- } \\
\text { tos con calidad aceptable. } \\
\text { Conocen su capacidad de produc- } \\
\text { ción. }\end{array}$ \\
\hline
\end{tabular}




\begin{tabular}{|c|c|c|}
\hline $\begin{array}{l}\text { Mercadotecnia orienta- } \\
\text { da al cliente }\end{array}$ & $\begin{array}{l}\text { Pelham (1997); Lin (1998), García } \\
\text { (1998), Zapata (2001) } \\
\text { Schlesinger y Useche (2005), Coca } \\
\text { (2006), Coy, Shipley et al. (2007), } \\
\text { Piedrahita y Paz (2010), Curmei } \\
\text { et al. (2011), Lekmat et al. (2018), } \\
\text { Sánchez et al. (2019), Peterson y } \\
\text { Crittenden (2020) }\end{array}$ & $\begin{array}{l}\text { Conocen su mercado objetivo, pero } \\
\text { desconocen sus características } \\
\text { propias: edad, hábitos de consu- } \\
\text { mo, etc. Buscan la satisfacción del } \\
\text { cliente. Se deben establecer rela- } \\
\text { ciones que perduren en el tiempo. } \\
\text { Usan la segmentación de mercado. } \\
\text { Buscan el valor para el cliente. }\end{array}$ \\
\hline Producto & $\begin{array}{l}\text { Rojas y Briceño (2006), Coy et al. } \\
\text { (2007), Wai-Sum (2005), Curmei } \\
\text { et al. (2011) }\end{array}$ & $\begin{array}{l}\text { Énfasis en la calidad y sus ca- } \\
\text { racterísticas. Se debe considerar } \\
\text { como estratégico la innovación. }\end{array}$ \\
\hline Precio & $\begin{array}{l}\text { Rojas y Briceño (2006), Alcánta- } \\
\text { ra et al. (2013), Wai-Sum (2005), } \\
\text { Curmei et al. (2011), Saavedra y } \\
\text { Saavedra (2014) }\end{array}$ & $\begin{array}{l}\text { Uso del método de costo más un } \\
\text { porcentaje de ganancias. } \\
\text { Seis de cada diez fijan su precio } \\
\text { con base en el costo. Se debe in- } \\
\text { cluir el enfoque de innovación. }\end{array}$ \\
\hline Plaza (distribución) & $\begin{array}{l}\text { Rojas y Briceño (2006), Alcánta- } \\
\text { ra et al. (2013), Wai-Sum (2005), } \\
\text { Curmei et al. (2011) }\end{array}$ & $\begin{array}{l}\text { Directa al consumidor, dispues- } \\
\text { to a sumarse a la innovación de } \\
\text { acuerdo con los cambios en los } \\
\text { mercados. }\end{array}$ \\
\hline Promoción & $\begin{array}{l}\text { Rojas y Briceño (2006), Alcántara } \\
\text { et al. (2013), Curmei et al. (2011), } \\
\text { Wai-Sum (2005) }\end{array}$ & $\begin{array}{l}\text { Las PYME no hacen promoción, } \\
\text { sólo venta personal, sin embargo, } \\
\text { debe innovar en esta práctica. }\end{array}$ \\
\hline $\begin{array}{l}\text { Conocimiento de la } \\
\text { competencia }\end{array}$ & $\begin{array}{l}\text { Huck y McEwen (1981), Coca } \\
(2006) \text {, } \\
\text { Lekmat et al. (2018) }\end{array}$ & $\begin{array}{l}\text { Se reconoce la importancia de } \\
\text { conocer a los competidores, pero } \\
\text { no se aplica. Aunque investigacio- } \\
\text { nes recientes refieren que más del } \\
50 \% \text { si lo aplican. }\end{array}$ \\
\hline $\begin{array}{l}\text { Estrategias mercadoló- } \\
\text { gicas (generales) }\end{array}$ & $\begin{array}{l}\text { García y Álvarez (1996), } \\
\text { Luk (1996), Carson et al. (2004), } \\
\text { Curmei et al. (2011), Saavedra } \\
\text { y Saavedra (2014), Mone et al. } \\
(2013)\end{array}$ & $\begin{array}{l}\text { Predomina la venta personal di- } \\
\text { recta. Aplican estrategias que } \\
\text { conduzcan la generación de ven- } \\
\text { tas. Las técnicas de marketing } \\
\text { conectan a las PYME con el mer- } \\
\text { cado. Siguen una estrategia de } \\
\text { segmentación. Buscan establecer } \\
\text { redes de comercialización. }\end{array}$ \\
\hline Servicio post venta & $\begin{array}{l}\text { Warren y Hutchinson (2000) } \\
\text { Wai-Sum (2005), Monferrer (2013) }\end{array}$ & $\begin{array}{l}\text { Pocas veces las PYME implemen- } \\
\text { tan esta técnica. Este servicio va } \\
\text { aunado a la facilidad que deben } \\
\text { tener los clientes para realizar re- } \\
\text { clamaciones, sobre el producto o } \\
\text { servicio. }\end{array}$ \\
\hline
\end{tabular}




\begin{tabular}{|l|l|l|}
\hline $\begin{array}{l}\text { Innovación } \\
\text { Marketing innovador }\end{array}$ & $\begin{array}{l}\text { Verhees y Meulenberg (2004), } \\
\text { O’Dwyer et al. (2009), Curmei et } \\
\text { al. (2011), Saavedra y Saavedra } \\
\text { (2014), Lekmat et al. (2018), Pe- } \\
\text { tkovska et al. (2018), Sánchez et } \\
\text { al. (2019), Mehran y Zeinab (2020) }\end{array}$ & $\begin{array}{l}\text { Mejora el desempeño dado que } \\
\text { promueve la creación de valor. } \\
\text { Solo una tercera parte realiza in- } \\
\text { novación en el producto, proceso } \\
\text { organización. Cuentan con ca- } \\
\text { pacidad para introducir con éxito } \\
\text { nuevos productos. }\end{array}$ \\
\hline Pronóstico de ventas & $\begin{array}{l}\text { Wai-Sum (2005), Curmei et al. } \\
\text { (2011), Saavedra y Saavedra } \\
(2014) \text { Solo la cuarta parte cumplió su } \\
\text { meta de ventas. La función de } \\
\text { marketing en las PYME garantiza } \\
\text { que alcance sus objetivos. }\end{array}$ \\
\hline Marca & $\begin{array}{l}\text { Wai-Sum (2005), Moferrer (2013), } \\
\text { Saavedra y Saavedra (2014) }\end{array}$ & $\begin{array}{l}\text { Desarrollo de marca propia. Se } \\
\text { debe buscar la diferenciación a } \\
\text { través de la marca. Menos de la } \\
\text { mitad de las PYME tiene registra- } \\
\text { da su marca. }\end{array}$ \\
\hline
\end{tabular}

Fuente: Elaboración propia con base en los autores citados.

Así pues, con base en la revisión anterior se concluye que el área de comercialización en las PYME incluye las siguientes prácticas: plan de mercado, estrategias comerciales, conocimiento de los competidores, determinación de precios, productos nuevos, marketing, servicio al cliente, fuerza de ventas y sistema de distribución.

\subsection{La competitividad en las PYME}

La competitividad en las PYME ha sido estudiada y conceptualizada desde diversos ángulos, así pues, de acuerdo con Saavedra (2014b, p. 20) "la competitividad de una empresa depende de la productividad, la rentabilidad, la posición competitiva, la posición en el mercado interno y externo, las relaciones interempresariales, el sector y la infraestructura regional y el entorno macroeconómico". Este concepto se deriva del modelo sistémico de competitividad de la CEPAL, que afirma que la competitividad empresarial se genera con la articulación de áreas que se encuentran en cuatro niveles o estratos que se presentan separados para un mejor estudio y análisis, estos son (Esser et al., 1996, Saavedra, 2014b): 1) Nivel macro: Incluye el entorno económico conformado principalmente por las políticas presupuestal, monetaria, fiscal, cambiaria, comercial, y de competencia; 2) Nivel meta: Se traduce en Factores socioculturales, que incluye principalmente la escala de valores de la sociedad, es decir la forma en que se encuentra organizada y es capaz de encontrar consensos a través del uso de estrategias y política; 3) Nivel meso: Identificado como el desarrollo regional, que se encuentra determinado principalmente con las Políticas de infraestructura y equipamiento, de articulación productiva (conglomerados y clúster), de articulación territorial (entre territorios, comunidades y ciudades), de capital logístico (transporte multimodal, telecomunicaciones y tecnología), de capital intelectual (innovación), de importaciones y exportaciones; y por último el 4) Nivel micro: Factores internos de la empresa, el cual se puede medir con MAPA de competitividad del BID, que se describe en el apartado 2.1.

Si la competitividad de las PYME se midiera únicamente con la productividad, se evidenciaría la gran desventaja que muestra frente a la gran empresa, pues de acuerdo con Dini y Stumpo (2018) en México las empresas de tamaño micro alcanzan sólo 
el $8 \%$ de productividad, las de tamaño pequeño alcanzan $24 \%$ de productividad y las medianas alcanzan $48 \%$ de productividad (tomando como referencia la empresa grande $=100 \%$ ); el rezago es aún más grande si se compara con los países europeos donde la brecha de productividad de las empresas pequeñas con la empresa grande es mucho menor, como en caso de Italia donde las empresas de tamaño micro alcanzan sólo el $25 \%$ de productividad, las de tamaño pequeño alcanzan $62 \%$ de productividad y las medianas alcanzan $84 \%$ de productividad.

Los estudiosos de la competitividad empresarial han propuesto modelos e indicadores para determinar la competitividad de las PYME, así pues, la OECD (1992) señala que los elementos que impulsan la competitividad son a) la eficiente administración de la producción e inventarios, b) la integración del plan de comercialización, I+D, diseño, ingeniería y producción, c) la combinación de I+D propia y la que se genera en las Instituciones de Educación Superior y Centros de investigación, d) la adaptación a los cambios en la demanda y a la evolución de los mercados, y e) la capacidad para integrarse en la cadena de valor. Por su parte, la Comisión Económica para América Latina, Cepal (1999) propuso analizar la competitividad empresarial con un único indicador que es la capacidad de exportación, lo cual permite conocer que países compiten en que industrias, así también, propone analizar la competitividad considerando nueve factores: fortaleza económica, internacionalización, gobierno e instituciones, finanzas, infraestructura, gestión empresarial, ciencia y tecnología, recursos humanos y medio ambiente.

Por su parte, Gómez (2002) sugiere que existen factores endógenos (tecnología, recursos humanos, gestión gerencial y comercialización) y exógenos (contexto económico y la conformación del mercado), que influyen la competitividad de las PYME. Otros más como Quiroga (2003), Solleiro y Castañon (2005), Rubio y Aragón (2006), De la Cruz et al. (2006), Martínez et al. (2009), Rohvein et al. (2012), Barrios et al. (2019) han propuesto modelos que van desde los matemáticos, hasta los que consideran los recursos y capacidades de las empresas, así como los enfocados en la cadena de valor y gestión de procesos, variando en el número y conceptualización de variables utilizadas. Estudios posteriores, como los de Rodríguez y Fernández (2006), Morales y Castellanos (2007), Montoya et al. (2010), Cano et al. (2013), Narváez et al. (2013) e Ibarra et al. (2017), han aplicado tanto modelos sistémicos, como internos para determinar la competitividad de las PYME.

\subsection{Las prácticas de comercialización y su relación con la competitividad}

La importancia de estudiar las prácticas de comercialización radica en que sólo un marketing efectivo podría ayudar a las PYME a ganar ventaja competitiva a través de un rendimiento superior, logrado con estrategias de marketing competitivo (Yan \& Chew, 2011). Así pues, diversos autores han analizado la capacidad de Marketing, como un factor importante para el impulso de la competitividad en las pequeñas empresas entre estos se encuentran: Huck y McEwen (1991), García y Álvarez (1996), Luk (1996), Pelham (1997), Lin (1998), Warren y Hutchinson (2000), Pelham (2000), Rubio y Aragón (2002), Verhees y Meulenberg (2004), Siu et al. (2004), Aragón y Rubio (2005), Piedrahita y Paz (2010), Franco et al. (2014), Lekmat et al. (2018) y JoensuuSalo et al. (2018), quienes han encontrado una relación significativa entre las prácticas de marketing y el desempeño de las PYME. Por su parte, Pil y Holwelg (2003), refieren que las PYME cuentan con ventajas como: la cercanía con sus clientes y el estableci- 
miento de redes, por lo que es capaz de obtener una ventaja competitiva al servir mejor a su mercado.

Así también, Sánchez et al. (2019), en una muestra de 400 PYME de la industria del mueble en Jalisco encontraron que el marketing innovador crea valor para el cliente, estableciendo también que este a su vez aumenta la competitividad de la empresa, puesto que conduce a la optimización de costos y al mejor uso de las capacidades tecnológicas. Más, sin embargo, Mone et al. (2013) dan cuenta de que son escasas las PYME que establecen una medición del desempeño del área de comercialización, aunque lo consideran importante.

En este mismo sentido, Gamal et al. (2020), analizaron la orientación al mercado (que incluyó, la orientación al cliente, la orientación a la competencia y la coordinación interfuncional) en 393 PYME de Arabia Saudita, encontrando una relación positiva significativa entre esta y el rendimiento, lo que se debería a que las PYME orientadas al mercado pueden satisfacer mejor las necesidades y expectativas de los clientes a través de la creación de productos y servicios innovadores, utilizando sus capacidades para aprovechar las oportunidades actuales, haciendo frente a sus competidores y creando así una ventaja competitiva sostenible, tal como lo comprobaron García et al. (2014).

Por su parte, Cuevas et al. (2020) estudiaron 341 empresas PYME en el estado de Guanajuato, México, estableciendo la existencia de una relación positiva significativa entre la innovación en marketing y el rendimiento de las empresas, considerando en el primero, aspectos tales como: métodos de comercialización, diseño del producto, modificaciones de envases, cambios significativos en el producto para incursionar en nuevos segmentos, nuevos sistemas de distribución, nuevos conceptos y medios de promoción, lanzamiento de productos o servicios en nuevos segmentos, nuevas estrategias para incentivar ventas, nuevos métodos de fijación de precios.

La investigación de Curmei et al. (2011), refiere que entre los factores que influyen en la competitividad de las PYME se puede incluir, la innovación de alto impacto que debe considerarse desde la planeación estratégica de marketing como elemento central en la segmentación, posicionamiento y dimensionamiento de la mezcla de marketing, buscando obtener ventajas competitivas.

Los estudios antecedentes han probado la relación existente entre las prácticas de Comercialización y la competitividad de las PYME (ya sea que se haya conceptualizado como rendimiento, productividad, ventaja competitiva, desempeño) lo que permite fundamentar esta investigación y formular la hipótesis siguiente:

Hi $_{1}$ : Existe relación entre las prácticas de Comercialización y la competitividad de las PYME en la Ciudad de México.

De la que se desprende la siguiente, hipótesis nula:

$H_{1}$ : No existe relación entre las prácticas de Comercialización y la competitividad de las PYME en la Ciudad de México.

Así también, se plantearon también hipótesis específicas, con el fin de determinar esta misma relación de acuerdo con el tamaño y el sector de las PYME. 
$\mathbf{H i}_{2}$ : Existe relación entre la competitividad de las micro empresas, pequeñas y medianas empresas y las prácticas de Comercialización

Ho$_{2}$ : No existe relación entre la competitividad de las micro empresas, pequeñas y medianas empresas y las prácticas de Comercialización

$\mathbf{H i}_{3}$ : No existe relación entre la competitividad de la empresa industrial, comercial y de servicios y las prácticas de Comercialización

Ho $_{3}$ : No existe relación entre la competitividad de la empresa industrial, comercial y de servicios y las prácticas de Comercialización

\section{Método}

Se realizó un estudio descriptivo con alcance correlacional donde se identificaron las prácticas de comercialización que utilizan las PYME y se estableció la relación que existe entre la competitividad interna y las referidas prácticas. La recolección de datos se realizó aplicando un cuestionario directo estructurado denominado Mapa de Competitividad del BID, el cual se describe en el apartado 2.1. Se determinó una muestra de 400 empresas correspondientes a las 16 alcaldías de la Ciudad de México, sobre una población de 382,056 unidades económicas (INEGI, 2010), con un nivel de confianza de $95 \%$ y un máximo de error de 5\%. El cuestionario de aplicó de manera personalizada a los empresarios que aceptaron participar en este estudio voluntariamente.

La comprobación estadística de las hipótesis se realizó aplicando el método de chi cuadrado, con un nivel de confianza de 95\% y un error de 5\%. Por lo tanto, no se puede rechazar la hipótesis cuando $p>0.05$. También se aplicó la prueba de correlación de Spearman.

\subsection{Mapa de competitividad del BID}

El mapa de competitividad $^{2}$ fue desarrollado por Banco Interamericano de Desarrollo (BID) y adaptado por la Cámara de Comercio de Medellín y Antioquía, para realizar un diagnóstico empresarial que permitiera conocer las fortalezas y debilidades de las PYME (Martínez \& Álvarez, 2006), para efectos de esta investigación se usa el instrumento adaptado por Saavedra (2014b). Este instrumento consta de un cuestionario que contiene145 preguntas que se agrupan en ocho áreas, cuyas respuestas se encuentran en una escala de Likert del 1 al 5, donde 1 corresponde a no cumple y 5 a cumple totalmente. Las ocho áreas que contiene el mapa son: Planeación estratégica, Producción y operaciones, Aseguramiento de la calidad, Comercialización, Contabilidad y finanzas, Capital humano, Gestión ambiental y Sistemas de Información.

Por otra parte, Saavedra (2014b) estableció la evaluación del nivel de competitividad global y por áreas de acuerdo con el cumplimiento que marquen las respuestas recogidas por los empresarios, en los siguientes rangos:

De 0-20\%: Muy baja competitividad

De $21 \%-40 \%$ : Baja competitividad

De 41\%-60\%: Mediana competitividad

2 Se conoce como mapa puesto que, al obtener una evaluación de cada área de la empresa, se presenta el resultado en un esquema en forma de araña. 
De $61 \%$ a $80 \%$ : Alta competitividad

De $81 \%$ a $100 \%$ : Muy alta competitividad

La confiabilidad del cuestionario, medida con el coeficiente Alfa de Cronbach fue de $91 \%$ que significa alta confiabilidad. En esta investigación se utilizó solamente los datos de competitividad del área Comercialización, dada la importancia de su estudio, a continuación, se definen las prácticas que la componen de acuerdo con la estructura del cuestionario Mapa de competitividad del BID (Saavedra, 2014b y Saavedra \& Milla, 2017):

Plan de mercado. Plan de mercado anual y escrito donde se desarrollan las estrategias, mercadológicas con base en la estructura del mercado y la identificación de oportunidades, permite dirigir y coordinar el esfuerzo de mercadotecnia, necesario para el logro de objetivos de ventas y utilidades de la empresa (Kotler, 2001). Muy pocas PYME realizan un plan de mercado lo que limita la implementación de estrategias comerciales (Wai-Sum, 2005; Saavedra \& Saavedra, 2014).

Estrategias comerciales. Definición de su mercado objetivo, estrategias de penetración, posicionamiento y comercialización, así como conocer los segmentos del mercado para desarrollar estrategias comerciales de acuerdo con los objetivos que se quieren alcanzar. Se establecen tomando como base el pronóstico de ventas, estimando la cantidad de productos o servicios a colocar en un lugar y tiempo determinados (Longenecker et al., 2012). Las PYME realizan estrategias comerciales considerando que sirven al consumidor final (García \& Álvarez, 1996; Luk, 1996; Saavedra \& Saavedra, 2014).

Conocimiento de competidores. Disponer de información de sus competidores, como elemento fundamental para realizar una planeación efectiva de las estrategias de mercado; constantemente, la empresa debe establecer un benchmarking de la mezcla de mercadotecnia con sus competidores, con el fin de identificar ventajas o desventajas competitivas (Kotler, 2001). Las PYME reconocen la importancia de conocer a los competidores, pero pocas realizan esta función (Huck \& McEwen, 1991).

Determinación de precios. Determinación de precios con base en los costos totales. El precio es parte del valor que perciben los consumidores, por lo que una buena definición y estrategia de precio conllevan al éxito (Peñalosa et al. 2017). El precio es el componente más efectivo de las estrategias de marketing, porque tiene relación directa con las utilidades, supervivencia y crecimiento de la empresa puesto que impulsa las ventas (Sheetal et al., 2012; Schnarch, 2013). Las PYME principalmente determinan los precios con base en los costos más un porcentaje de ganancias (Rojas \& Briceño, 2006; Alcántara et al., 2013; Wai-Sum, 2005; Saavedra \& Saavedra, 2014).

Productos nuevos. Se debe determinar si los productos nuevos han generado ventas y utilidades importantes en la empresa. La clave del éxito para las PYME es la innovación mediante el desarrollo continuo del conocimiento que para lograr su posicionamiento en un entorno dinámico (O’Dwyer et al., 2009). Es así como la innovación viene a ser un aspecto fundamental en el proceso de comercialización, es importante innovar en referencia a los avances tecnológicos y a los gustos y preferencias de los clientes. Las PYME deben poner énfasis en la calidad y características de los productos que logren una diferenciación (Rojas \& Briceño, 2006; Coy et al., 2007; 
Wai-Sum, 2005); sin embargo, muy pocas realizan innovación de productos (Verhees \& Meulenberg, 2004; O’Dwyer et al., 2009; Saavedra \& Saavedra, 2014).

Marketing. Son todas las actividades que se realizan para facilitar y estimular intercambios entre distintos entes, entre las que se encuentran planear, fijar el precio, promover y distribuir los productos o prestar los servicios, buscando conocer las necesidades de los consumidores y la evaluación de las actividades de promoción para fomentar las ventas con el fin de alcanzar los objetivos de la empresa (Peñalosa et al., 2017). Las PYME pueden diseñar estrategias de Marketing orientadas a la producción (Rojas \& Briceño, 2006; Domínguez et al., 2012); o al cliente (Pelham, 1997; Lin, 1998; García, 1998; Zapata, 2001; Schlesinger \& Useche, 2005; Coy et al., 2007; Peterson \& Crittenden, 2020; Piedrahita \& Paz, 2010); sin embargo, muy pocas utilizan estrategias de promoción (Rojas \& Briceño, 2006; Alcántara, et al., 2013; Wai-Sum, 2005).

Servicio al cliente. Se refiere al conocimiento y satisfacción de las necesidades del cliente, así como, contar con catálogos de especificaciones técnicas de los productos. Franco et al. (2014) sugieren que un mal servicio de las PYME puede producir insatisfacción de sus clientes y afectar su imagen, su competitividad, dificultando la fidelización de los clientes. Por otra parte, muy pocas empresas del sector PYME implementan los servicios post venta (Warren \& Hutchinson, 2000; Wai-Sum, 2005).

Fuerza de ventas. Establecimiento de objetivos o cuotas de ventas para los vendedores, se debe procurar tener una fuerza de ventas capacitada, motivada y competente, con vínculo laboral en la empresa. La fuerza de ventas funciona como enlace entre la empresa y los clientes, para una administración eficiente de la fuerza de ventas se necesita considerar: Objetivos de ventas, estrategia, organización, volumen, promociones a vendedores (Kotler, 2001). Las PYME escasamente cumplen con sus metas de ventas (Wai-Sum, 2005, Saavedra \& Saavedra, 2014), debido a factores tanto internos como externos que afectan su desempeño.

Sistema de distribución. La distribución es el proceso que se realiza para lograr que los productos/servicios lleguen en el tiempo y lugar adecuado al consumidor final, es decir una distribución efectiva. Para llevar a cabo el proceso de distribución se debe decidir sobre el tipo de distribución: masiva, exclusiva o selectiva y el canal de distribución: directo o a través de canales que incluyen mayoristas, minoristas y agentes comerciales (Sheetal et al., 2012; Peñalosa et al., 2017). Las PYME generalmente utilizan la distribución directa al consumidor final (Rojas \& Briceño, 2006; Alcántara et al., 2013, Wai-Sum, 2005).

\subsection{Operacionalización de variables}

A continuación, se presenta la manera en que fueron operacionalizadas las variables de acuerdo con las hipótesis planteadas para esta investigación (tabla 2). 
Tabla 2. Operacionalización de variables

\begin{tabular}{|c|c|c|c|}
\hline Hipótesis & Variable 1 & Variable 2 & $\begin{array}{l}\text { Indicadores } \\
\text { Variable } 2\end{array}$ \\
\hline $\begin{array}{l}\text { Hi1: Existe relación } \\
\text { entre las prácticas de } \\
\text { Comercialización y la } \\
\text { competitividad de las } \\
\text { PYME en la Ciudad } \\
\text { de México. }\end{array}$ & $\begin{array}{l}\text { Competitividad } \\
\text { Se mide como el } \\
\text { promedio simple de } \\
\text { la competitividad } \\
\text { global, aplicando el } \\
\text { mapa de competiti- } \\
\text { vidad del BID, de las } \\
\text { empresas que confor- } \\
\text { man la muestra. }\end{array}$ & $\begin{array}{l}\text { Prácticas de Co- } \\
\text { mercialización }\end{array}$ & $\begin{array}{l}\text { Plan de mercado } \\
\text { Estrategias comerciales } \\
\text { Conocimiento de competi- } \\
\text { dores } \\
\text { Determinación de precios } \\
\text { Productos nuevos } \\
\text { Marketing } \\
\text { Servicio al cliente } \\
\text { Fuerza de ventas } \\
\text { Sistema de distribución }\end{array}$ \\
\hline $\begin{array}{l}\text { Hi2: Existe relación } \\
\text { entre la competitivi- } \\
\text { dad de las micro em- } \\
\text { presas, pequeñas y } \\
\text { medianas empresas } \\
\text { y las prácticas de Co- } \\
\text { mercialización }\end{array}$ & $\begin{array}{l}\text { Competitividad por } \\
\text { tamaño } \\
\text { Se mide como el pro- } \\
\text { medio simple de la } \\
\text { competitividad glo- } \\
\text { bal de las empresas } \\
\text { según el tamaño por } \\
\text { separado: } \\
\text { Micro } \\
\text { Pequeña } \\
\text { Mediana }\end{array}$ & $\begin{array}{l}\text { Prácticas de Co- } \\
\text { mercialización }\end{array}$ & $\begin{array}{l}\text { Plan de mercado } \\
\text { Estrategias comerciales } \\
\text { Conocimiento de competi- } \\
\text { dores } \\
\text { Determinación de precios } \\
\text { Productos nuevos } \\
\text { Marketing } \\
\text { Servicio al cliente } \\
\text { Fuerza de ventas } \\
\text { Sistema de distribución }\end{array}$ \\
\hline $\begin{array}{l}\text { Hi3: No existe rela- } \\
\text { ción entre la competi- } \\
\text { tividad de la empresa } \\
\text { industrial, comercial } \\
\text { y de servicios y las } \\
\text { prácticas de Comer- } \\
\text { cialización }\end{array}$ & $\begin{array}{l}\text { Competitividad por } \\
\text { sector } \\
\text { Se mide como el pro- } \\
\text { medio simple de la } \\
\text { competitividad glo- } \\
\text { bal de las empresas } \\
\text { según el sector por } \\
\text { separado: } \\
\text { Industria } \\
\text { Comercio } \\
\text { Servicio }\end{array}$ & $\begin{array}{l}\text { Prácticas de Co- } \\
\text { mercialización }\end{array}$ & $\begin{array}{l}\text { Plan de mercado } \\
\text { Estrategias comerciales } \\
\text { Conocimiento de competi- } \\
\text { dores } \\
\text { Determinación de precios } \\
\text { Productos nuevos } \\
\text { Marketing } \\
\text { Servicio al cliente } \\
\text { Fuerza de ventas } \\
\text { Sistema de distribución }\end{array}$ \\
\hline
\end{tabular}

Fuente: Elaboración propia. 


\section{La competitividad de las PYME en la Ciudad de México}

Este trabajo presenta la tercera parte del proyecto de investigación "La determinación de la competitividad de las PYME en el Distrito Federal ${ }^{3 "}$ en el cual se determinó la competitividad interna de las PYME considerando ocho áreas, por lo que en primer lugar (apartado 3.1), se presenta un resumen de los hallazgos preliminares de Saavedra (2014b) con el fin de contextualizar la presente investigación, en segundo lugar se presenta el análisis en interpretación de los hallazgos de la presente investigación (3.2).

\subsection{Hallazgos preliminares}

Estos hallazgos corresponden a la primera parte de la investigación en el que se analizó la competitividad de las PYME en todo su conjunto sin separar por áreas y fue resumido de Saavedra (2014b).

La muestra está conformada por 41 (10.3\%) empresas del sector industria, comercio 192 empresas $(48.0 \%$ ) y servicios 167 empresas (41.8\%), proporción bastante similar con las clasificación sectorial nacional de acuerdo con INEGI (2020); por tamaño, la conformación fue: 364 (91.0\%) microempresas, 29 (7.3\%) empresas pequeñas y sólo $7(1.8 \%)$ empresas medianas, como puede apreciarse predominan las empresas de tamaño micro (1-10 trabajadores) de los sectores comercio y servicios, coincidiendo con la estratificación nacional de acuerdo con INEGI (2020). La competitividad global promedio fue sólo $41.85 \%,{ }^{4}$ donde el $73.3 \%$ de las empresas son de muy baja o baja competitividad y sólo el 17\% de alta o muy alta competitividad (Saavedra, 2014b). Esta diferencia en el nivel de competitividad se debería a que en esta ciudad existen empresas con procesos productivos muy modernos que usan alta tecnología y mano de obra calificada capaces de exportar sus productos, y también empresas que se encuentran en la informalidad, con procesos artesanales de producción y condiciones precarias de trabajo, especialmente las que se encuentran en el sector rural (Gaceta Oficial del Distrito Federal, 2013). A pesar de la importancia de las PYME, en México solo el 4.3\% del PIB se usa para su financiamiento, lo cual muestra rezago si se compara con algunos países emergentes e industrializados con los que México compite o es socio. Así, se tiene el caso de Brasil donde este monto es del 9.6\%, en España el 19.6\% y en Estados Unidos el 29.4\% del PIB (Gaceta Oficial del Distrito Federal, 2013).

Los resultados de la competitividad global por áreas, muestran que la única área que logra rebasar el $50 \%$ de competitividad es el de aprovisionamiento, quedando todas las demás rezagados, donde el área de comercialización alcanza sólo el 45.5\% de competitividad; lo que resulta preocupante puesto que una gran parte son empresas de servicio y comercio que atienden principalmente al consumidor final, y si se considera que se trata de la competitividad interna de las PYME, lo que estaría mostrando la debilidad que muestran en la realización de sus procesos internos los cuales dependen directamente de sus propios recursos (figura 1).

3 El 5 de febrero de 2016, se publica en el Diario Oficial de la Federación el Acuerdo General del Pleno del Consejo de la Judicatura Federal por el que se cambia la denominación de Distrito Federal por Ciudad de México en todo su cuerpo normativo. Por esta razón en este trabajo se hace referencia a la Ciudad de México.

4 Se calculó como promedio de todas las empresas de la muestra. 
Figura 1. Mapa de Competitividad de la PYME de la Ciudad de México

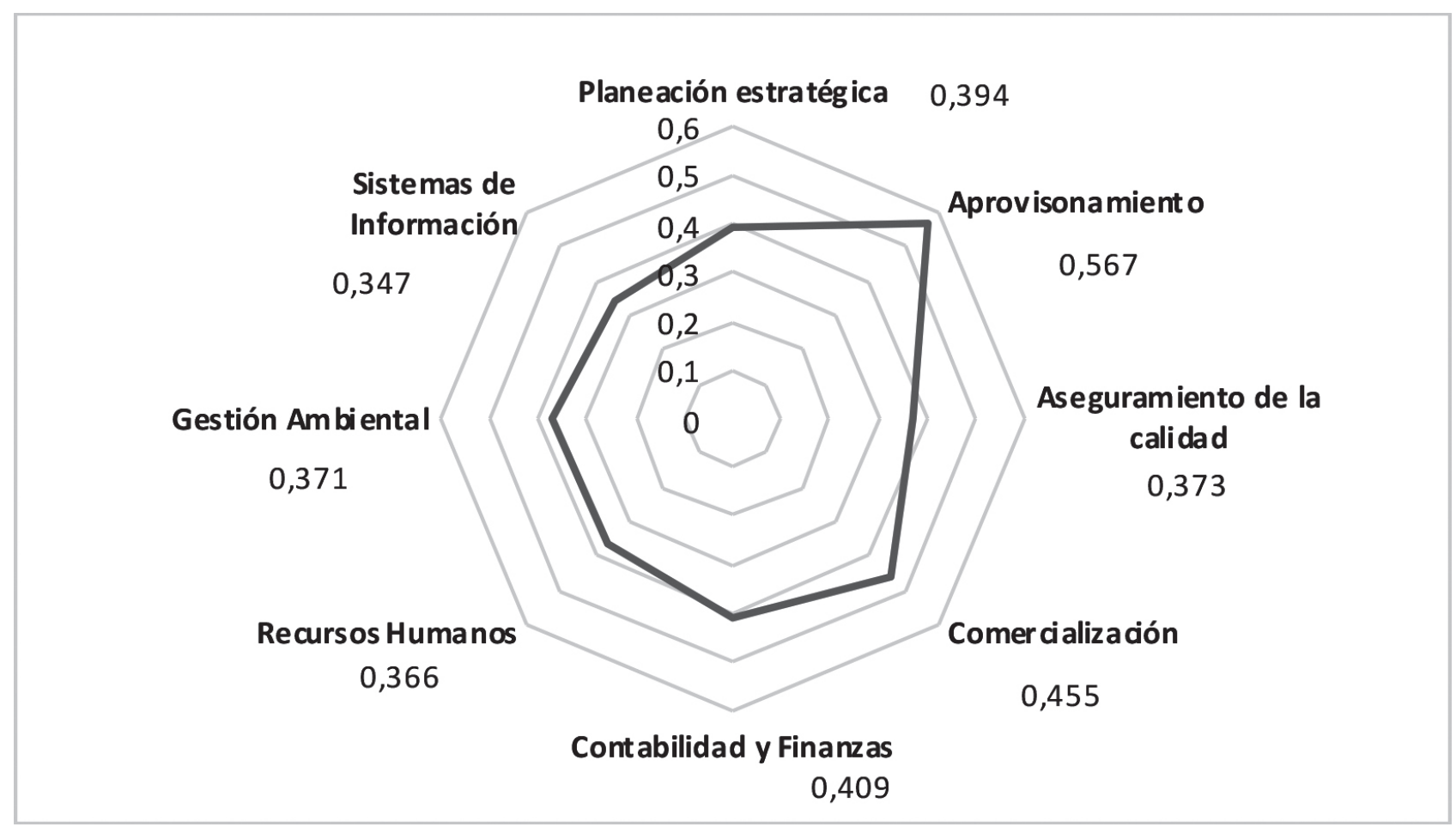

Fuente: Saavedra, 2014b

Los hallazgos de la competitividad por sector dan cuenta que, en la industria, sólo $36.6 \%$ cuentan con alta competitividad y cerca al $60 \%$ de baja competitividad. En cuanto a los sectores comercio, como servicios las tres cuartas partes de los negocios presentan baja y muy baja competitividad. Esta situación pone en riesgo la estabilidad de las PYME en esta ciudad, pues la misma aporta el 17.5\% del Producto Interno Bruto, con una tasa de crecimiento promedio superior a la nacional, lo que muestra un elevado dinamismo económico, con una economía enfocada al comercio y servicios (Secretaría de Desarrollo Económico, 2019). En esta ciudad, el turismo ha detonado el desarrollo de empresas de hospedajes, alimentos y bebidas y las oportunidades de inversión han impulsado el desarrollo de las industrias que necesitan alcanzar niveles elevados de competitividad que les permita permanecer en el largo plazo y seguir generando los empleos que se requieren (Gaceta Oficial del Distrito Federal, 2013). Sin embargo, este hallazgo permite saber que el sector industria presenta mayores niveles de competitividad que los sectores comercio y servicios, los cuales son predominantes en esta ciudad.

En lo que se refiere a la competitividad por tamaño de empresa, los resultados muestran que el $80.2 \%$ de las micro empresas tienen baja competitividad, mientras que en la pequeña empresa el $75.9 \%$ es de alta competitividad, en tanto que en la mediana empresa el $100 \%$ es de muy alta competitividad, lo que implica que la competitividad está relacionada con el tamaño de la empresa, lo cual resulta lógico pues una empresa de mayor tamaño cuenta con mayores recursos, tecnológicos, humanos, etc. que impulsan su competitividad. Estos hallazgos, implican que siendo la sexta que parte del PIB turístico nacional lo produce la Ciudad de México y una alta proporción de hoteles y restaurantes son micro y pequeñas empresas (Gaceta Oficial del Distrito Federal, 2013), se presenta como reto pendiente elevar la competitividad de las empre- 
sas de este sector, por la importancia que reviste no solo para la economía de la Ciudad de México, sino también de México.

\subsection{Las prácticas de comercialización y la competitividad en las PYME de la Ciudad de México}

En este apartado se presentan los hallazgos de la determinación de la competitividad del área de Comercialización, su relación con la competitividad global, por tamaño y por sector, éstos corresponden a la tercera parte de la investigación objeto de este artículo donde se analizan las prácticas del área de comercialización y su relación con la competitividad, considerando también el tamaño y el sector.

\subsubsection{Hallazgos y discusión del nivel de competitividad de las prácticas del área de comercialización}

A continuación, se presenta el nivel de competitividad alcanzado por cada una de las prácticas del área de Comercialización de las empresas analizadas (Tabla 3), es decir la competitividad medida de acuerdo con el nivel de uso de las prácticas en estas empresas. Como se puede apreciar en la tabla 3, las prácticas en las que predomina alta y muy alta competitividad son cuatro: determinación de precios $(69.5 \%)$, servicio al cliente (57.75\%), fuerza de ventas (54\%) y sistema de distribución $(70.75 \%$, las cuales podrían ser consideradas como prácticas básicas que deben realizar las PYME, siendo estos hallazgos coincidentes con lo señalado por Wai-Sum (2005), Saavedra y Saavedra (2014), Cohen (2017), Petkovska et al. (2018), los cuales refieren que las PYME solo realizan actividades de comercialización en referencia directa a las ventas, puesto que generalmente sirven al consumidor final. Así también, en los resultados mostrados en la tabla 3, destacan las prácticas del área de comercialización en los predomina baja y muy baja competitividad, son cinco: Plan de mercado (62.5\%), estrategias comerciales (52.75\%), conocimiento de los competidores $(64.75 \%)$, productos nuevos $(55.75 \%)$ y marketing (51.55\%), los cuales se consideran como prácticas avanzadas que tienen relación directa con la visión de largo plazo de las PYME (Sheetal et al., 2012); siendo coincidente este hallazgo con lo encontrado por Huck y McEwen (1991), García y Álvarez (1996), Luk (1996), Wai-Sum (2005), Rojas y Briceño (2006), Sheetal et al. (2012), Alcántara et al. (2013), Saavedra y Saavedra (2014), los cuales dan cuenta del poco o nulo uso de estas prácticas avanzadas en las PYME, lo que implica el predominio de la visión de corto plazo en las PYME estudiadas en la presente investigación. Por lo tanto, es necesario que estas empresas implementen prácticas avanzadas de comercialización, pues Mehran y Zeinab (2020), señalan que las prácticas tradicionales resultan insuficientes en un ambiente turbulento y de alta competencia como el actual. 
Tabla 3. Competitividad de las prácticas de comercialización (Porcentaje)

\begin{tabular}{|c|c|c|c|c|c|c|c|c|c|}
\hline 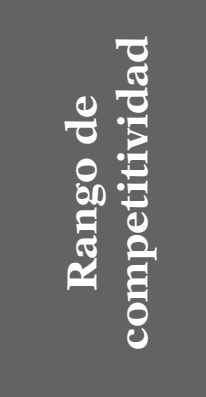 & 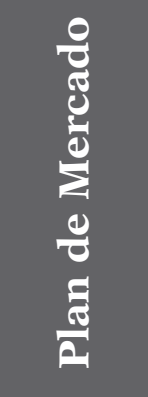 & 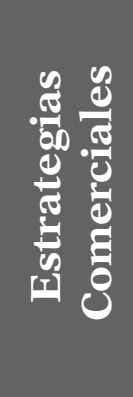 & 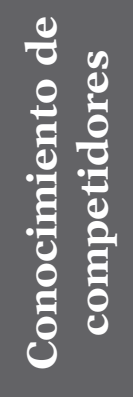 & 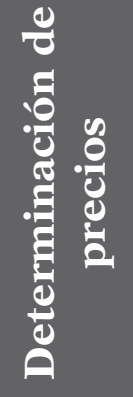 & 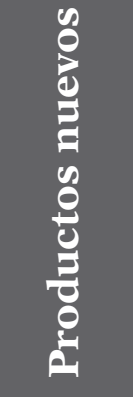 & 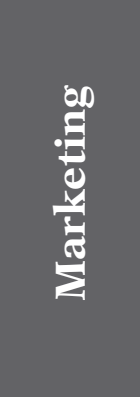 & 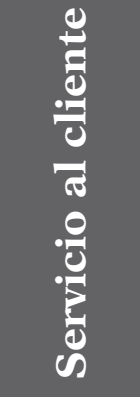 & 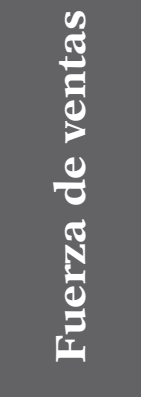 & 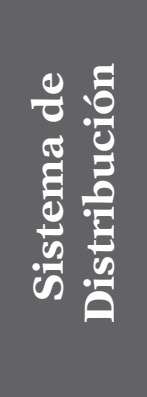 \\
\hline $\begin{array}{l}\text { Muy alta } \\
81-100 \%\end{array}$ & 18.75 & 12.75 & 8.25 & 56.75 & 7.25 & 12.75 & 22.50 & 25.00 & 57.75 \\
\hline $\begin{array}{l}\text { Alta } \\
61-80 \%\end{array}$ & 7.00 & 10.50 & 11.00 & 12.75 & 15.00 & 16.50 & 35.25 & 29.00 & 13.00 \\
\hline $\begin{array}{l}\text { Mediana } \\
41-60 \%\end{array}$ & 11.75 & 20.00 & 16.00 & 14.00 & 22.00 & 19.25 & 23.50 & 21.00 & 10.25 \\
\hline $\begin{array}{l}\text { Baja } \\
21-40 \%\end{array}$ & 9.75 & 34.25 & 33.25 & 4.75 & 38.00 & 27.50 & 15.50 & 11.25 & 4.50 \\
\hline $\begin{array}{l}\text { Muy baja } \\
0-20 \%\end{array}$ & 52.75 & 22.50 & 31.50 & 11.75 & 17.75 & 24.00 & 3.25 & 13.75 & 14.50 \\
\hline Total & 100.00 & 100.00 & 100.00 & 100.00 & 100.00 & 100.00 & 100.00 & 100.00 & 100.00 \\
\hline
\end{tabular}

Fuente: Elaboración propia.

\subsubsection{Hallazgos y discusión de la relación entre las prácticas del área de comercialización y la competitividad de las PYME}

En la tabla 4, los resultados muestran que todas las prácticas del área de comercialización tienen relación significativa con la competitividad de las PYME, lo que implica la importancia que tienen las prácticas de comercialización para impulsar la competitividad de las PYME, así también, la prueba de correlación de Spearman permitió determinar la fuerza de esta correlación, mostrando que todas las correlaciones son positivas así como, una alta correlación de ésta área con la competitividad global (68.2\%). Analizando individualmente los resultados de cada una de las prácticas las correlaciones más elevadas se dieron en fuerza de ventas (64.7\%), marketing (61.3\%), servicio al cliente $(53.6 \%)$ y plan de mercado (5.16\%), lo que implica la importancia del implementar el uso de estas prácticas para impulsar la competitividad de las PYME. Estos hallazgos son coincidentes con los estudios realizados por: Huck y McEwen (1991), Luk (1996), Pelham (1997), Lin (1998), Warren y Hutchinson (2000), Pelham (2000), Rubio y Aragón (2002), Verhees y Meulenberg (2004), Siu et al. (2004), Aragón y Rubio (2005), Piedrahita y Paz (2010), Franco et al. (2014), Lekmat et al. (2018), y Joensuu-Salo et al. (2018), quienes han encontrado que existe relación entre las prácticas del área de comercialización y la competitividad de las PYME. De acuerdo con los resultados anteriores, se rechaza la hipótesis nula (Ho1) por lo que se concluye que existe relación entre la competitividad y todas las prácticas del área de comercialización. 


\section{Tabla 4. Relación entre la competitividad global y las prácticas del área de comercialización}

\begin{tabular}{|l|c|c|}
\hline \multicolumn{1}{|c|}{ Factor } & Valor de p & $\begin{array}{c}\text { Correlación de } \\
\text { Spearman }\end{array}$ \\
\hline Plan de mercado & .000 & 0.516 \\
\hline Estrategias Comerciales & .000 & 0.441 \\
\hline Conocimiento de competidores & .000 & 0.473 \\
\hline Determinación de precios & .000 & 0.426 \\
\hline Productos nuevos & .000 & 0.409 \\
\hline Marketing & .000 & 0.613 \\
\hline Servicio al cliente & .000 & 0.536 \\
\hline Fuerza de ventas & .000 & 0.647 \\
\hline Sistema de distribución & .000 & 0.411 \\
\hline $\begin{array}{l}\text { Competitividad global y competitividad del área de } \\
\text { comercialización }\end{array}$ & .000 & 0.682 \\
\hline
\end{tabular}

Fuente: Elaboración propia.

Nota: Se rechaza Ho si $p \geq 0.05$

\subsubsection{Hallazgos y discusión de la relación entre la competitividad por tamaño de la empresa y las prácticas del área de comercialización}

En la tabla 5, se muestra un resumen del resultado de la prueba de chi cuadrado ( $p=$ significancia estadística) y la prueba de correlación de Spearman, de las prácticas de mercadotecnia y la competitividad global de acuerdo con el tamaño de las empresas. Como se puede observar, en el caso de las empresas de tamaño micro y pequeña, la importancia que tienen las prácticas plan de mercado (con una correlación de $41 \%$ y $49 \%$ respectivamente), productos nuevos (61\% y 67\% de correlación), marketing (con $33 \%$ y $41 \%$ de correlación), servicio al cliente (con $54 \%$ y $57 \%$ de correlación) y fuerza de ventas ( $42 \%$ y $48 \%$ de correlación), en la competitividad de estas empresas, esto se debería a que al ser prácticas avanzadas de mercadotecnia le permiten al empresario contar con una visión de largo plazo, estar constantemente innovando, generar estrategias de marketing para mantenerse en el mercado y mejorar el servicio al cliente con una fuerza de ventas capacitada, generando así ventajas competitivas. Siendo este hallazgo coincidente con lo que señalan Yan y Chew (2011) y Sheetal et al. (2012) en el sentido de que solo las estrategias de marketing competitivo pueden ayudar a las PYME a ganar ventaja competitiva y por consiguiente un rendimiento superior, esto cobra relevancia su se considera que es necesario incrementar la productividad de las micro y pequeñas empresas puesto que la brecha con respecto a la empresa grande es muy amplia (Dini \& Stumpo, 2018). Con relación a la empresa mediana, los resultados no mostraron relación de la competitividad con las prácticas de comercialización, debido a que, al ser empresas de mayor tamaño, son más estructuradas y existen otros 
factores que inciden en la competitividad de las mismas. Con los resultados anteriores se rechaza parcialmente Ho2 y se concluye que existe relación entre la competitividad de las empresas de tamaño micro y pequeña y las prácticas de comercialización que implican una visión de largo plazo por parte de los empresarios y por lo tanto podrían asegurar la permanencia de las PYME que las implementan.

\section{Tabla 5. Relación entre la competitividad por tamaño y las prácticas del área de comercialización}

\begin{tabular}{|c|c|c|c|c|c|c|}
\hline $\begin{array}{c}\text { Competitividad global y prácticas } \\
\text { del área de comercialización/ } \\
\text { Tamaño }\end{array}$ & 율 & 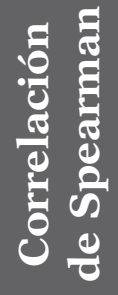 & 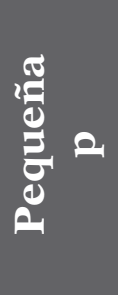 & 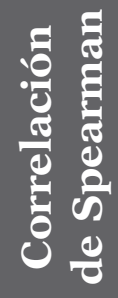 & 宽 & 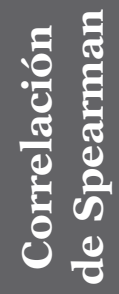 \\
\hline Plan de mercado & .000 & 0.412 & .000 & 0.492 & .214 & 0.076 \\
\hline Estrategias Comerciales & .139 & 0.103 & .305 & 0.079 & .569 & 0.070 \\
\hline $\begin{array}{l}\text { Conocimiento } \\
\text { de competidores }\end{array}$ & .456 & 0.040 & .559 & 0.018 & .732 & 0.057 \\
\hline Determinación de precios & .837 & 0.000 & .849 & 0.018 & .166 & 0.043 \\
\hline Productos nuevos & .034 & 0.512 & .009 & 0.673 & 650 & 0.066 \\
\hline Marketing & .001 & 0.334 & .001 & 0.415 & .300 & 0.010 \\
\hline Servicio al cliente & .000 & 0.547 & .002 & 0.571 & .531 & 0.075 \\
\hline Fuerza de ventas & .000 & 0.422 & .001 & 0.480 & .348 & 0.086 \\
\hline Sistema de distribución & .730 & 0.021 & .476 & 0.039 & 672 & 0.041 \\
\hline
\end{tabular}

Fuente: Elaboración propia.

Nota: Se rechaza Ho si $\mathrm{p} \geq 0.05$

\subsubsection{Hallazgos y discusión de la relación entre la competitividad por sector de la empresa y las prácticas del área de comercialización}

Los resultados de la tabla 6, muestran se muestra un resumen del resultado de la prueba de chi cuadrado ( $p=$ significancia estadística) y la prueba de correlación de Spearman, de las prácticas de mercadotecnia y la competitividad global de acuerdo con el sector al que pertenecen las empresas. Como se puede observar, existe relación de sólo cuatro prácticas del área de comercialización (Conocimiento de competidores, $36 \%$ de correlación; determinación de precios, 44\% de correlación; Marketing, 37\% de correlación y Servicio al cliente, 39\% de correlación) y la competitividad de las PYME industriales, este resultado se debería a que el sector industrial no sirve al consumidor final directamente, por un lado, y por el otro, en el sector industrial existen otros factores que impulsan su competitividad como los sistemas de calidad, la mano de obra calificada y la tecnología y no tanto las prácticas de comercialización. Por otra parte, 
se observa que en los sectores comercio y servicios, son importantes para impulsar la competitividad, siete prácticas de comercialización: plan de mercado, estrategias comerciales, conocimiento de los competidores, productos nuevos, servicio al cliente, fuerza de ventas y sistemas de distribución, mostrando con esto la importancia de contar con una visión de largo plazo, generar estrategias que impulsen la ventaja competitiva, prestar atención a las estrategias de los competidores, innovar contantemente, y poner al cliente en primer lugar, conociendo sus necesidades y haciéndole llegar los productos/servicios que requiere oportunamente y con las especificaciones pactadas, brindándole asesoría en todo momento; toda vez que las PYME de estos sectores sirven principalmente al consumidor final. Puesto que no se pudo establecer relación significativa de todas las prácticas que utilizan los sectores con la competitividad global, se rechaza parcialmente $\mathrm{Ho}_{3}$.

\section{Tabla 6. Relación entre la competitividad por sector y las prácticas del} área de comercialización

\begin{tabular}{|c|c|c|c|c|c|c|}
\hline $\begin{array}{l}\text { Competitividad global y Prácticas del } \\
\text { área de Comercialización / Sector }\end{array}$ & 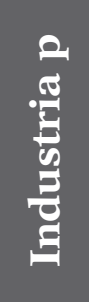 & 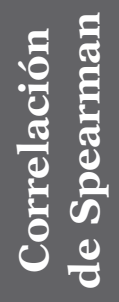 & $\begin{array}{l}\text { ㅇ } \\
0 \\
0 \\
0 \\
0 \\
0 \\
0 \\
0\end{array}$ & 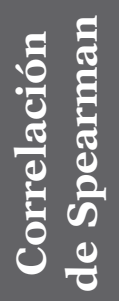 & $\begin{array}{l}0 \\
0 \\
0 \\
0 \\
0 \\
0 \\
0 \\
0\end{array}$ & 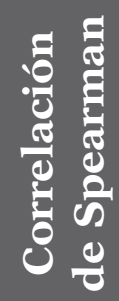 \\
\hline Plan de mercado & .357 & 0.088 & .002 & 0.491 & .016 & 0.400 \\
\hline Estrategias Comerciales & .647 & 0.028 & .016 & 0.366 & .016 & 0.345 \\
\hline Conocimiento de competidores & .032 & 0.360 & .032 & 0.450 & .011 & 0.511 \\
\hline Determinación de precios & .033 & 0.440 & .112 & 0.081 & .516 & 0.078 \\
\hline Productos nuevos & .783 & 0.023 & .002 & 0.328 & .009 & 0.459 \\
\hline Marketing & .041 & 0.371 & .920 & 0.019 & .113 & 0.089 \\
\hline Servicio al cliente & .022 & 0.391 & .005 & 0.597 & .018 & 0.613 \\
\hline Fuerza de ventas & .378 & 0.066 & .006 & 0.356 & .008 & 0.417 \\
\hline Sistema de distribución & .226 & 0.011 & .001 & 0.317 & .000 & 0.305 \\
\hline
\end{tabular}

Nota: Se rechaza Ho si $\mathrm{p} \geq 0.05$

\section{Conclusiones}

Los resultados de este trabajo permiten conocer que las PYME, solo utilizan prácticas básicas de comercialización que tienen relación con la determinación de precios basados en costos, servicio a clientes, fuerza de ventas y sistema de distribución, los cual muestra la visión de corto plazo que tiene el empresario por lo que no realiza planes de mercado y ni diseña estrategias comerciales, no conoce a sus competidores ni está innovando constantemente, no realiza actividades de promoción, para impulsar sus ventas, mantenerse y ampliar su mercado, lo que trae como resultado una baja competitividad que se muestra en el indicador de competitividad global de este factor 
que sólo alcanza $45.5 \%$ y en el bajo nivel de competitividad que tienen estas prácticas avanzados al no ser utilizadas por las PYME. Lo anterior, resulta preocupante toda vez que todas las prácticas de comercialización se encuentran relacionadas con la competitividad global de las empresas, por lo que es necesario fomentar el uso de las prácticas de comercialización avanzadas, para lo cual es necesario un cambio de visión del empresario hacia el largo plazo, que le permita sobrevivir y permanecer. Las empresas micro y pequeñas son las que más dependen que las prácticas de comercialización para impulsar su competitividad, pues la implementación de estas puede ayudarlas a alcanzar ventajas competitivas que les permita permanecer en el mercado. Los sectores comercio y servicios son los que más dependen de las prácticas de comercialización para impulsar su competitividad, debido a que existe gran competencia en estos sectores por su predominancia en la Ciudad de México, y también dado que sirven al consumidor final. Así pues, estos hallazgos plantean un reto a asumir y es el que los empresarios comprendan la importancia de utilizar sus recursos y capacidades en la implementación de las prácticas de comercialización, dada la importante relación que estos muestran con la competitividad que le genera rentabilidad en el largo plazo, lo cual a su vez se traduce en permanencia en el mercado.

El aporte principal de esta investigación es el conocimiento de las prácticas de comercialización que utilizan las PYME y el establecimiento de una relación significativa entre éstas y la competitividad; la limitación principal es el uso de una muestra de sujetos voluntarios, lo cual no permite la generalización de los hallazgos a toda la población.

\section{Referencias}

Alcántara, R., Goytortúa, C., \& Vega, A. (2013). Prácticas de mercadotecnia en las microempresas del sector comercial de la ciudad de Pachuca, Hidalgo: Un análisis y propuesta para apoyar su crecimiento. XVIII Congreso Internacional de Contaduría, Administración e Informática. Octubre-2-4, Ciudad Universitaria, México, D.F. México.

Aragón A., \& Rubio A. (2005). Factores explicativos del éxito competitivo: El caso de las PYMES del estado de Veracruz. Revista Contaduría y Administración, 216, 35-69, mayo-agosto.

Barrios, K. del C., Contreras, J., \& y Olivero, E. (2019). La gestión por procesos en las Pymes de Barranquilla: Factor Diferenciador de la Competitividad Organizacional. Información Tecnológica, 30(2), 57-72. http://dx.doi.org/10.4067/S0718-07642019000200103

Bretcu, A. (2014). Marketing Controlling and Price Policy for SMEs. Analele Universitatii "Eftimie Murgu" Resita. Fascicola II. Studii Economice, 34-41.

Bocconcelli, R., Cioppi, M., Fortezza, F., Francioni, B., Pagano, A., Savelli, E., \& Splendiani, S. (2018). SMEs and Marketing: A Systematic Literature Review. International Journal of Management Reviews, 20(2), 227-254. https://doi.org/10.1111/ijmr.12128

Cano, M., Olivera, D., Balderrabano, J., \& Pérez, G. (2013). Rentabilidad y competitividad en la PYME. Ciencia Administrativa, 2, 80-86.

Carson, D., Gilmore, A., \& Rocks, S. (2004). SME marketing networking: a strategic approach. Strategic Change, 13, 369-382. https://doi.org/10.1002/jsc.695

Cepeda, S., Velázquez, L., \& Marín, B. (2017). Análisis evaluativo a los procesos de marketing en la internacionalización de las pequeñas y medianas empresas de alimentos de Medellín. Estudios Gerenciales, 33 (271-280). https://doi.org/10.1016/j.estger.2017.06.006

Coca, M. (2006). El concepto de Marketing: Pasado y presente. Perspectivas, 9 (18), 41-72.

Cohen, J. (2017). Improving Marketing Knowledge among Israeli SMEs using Metaphor and Storyline-Based Intervention. Middle East Journal of Business, 12(3)10-19. https://doi.org/10.5742/MEJB.2018.93184 
Comisión Económica para América Latina (CEPAL) (1999). Sistema para analizar el crecimiento del comercio internacional (Magic). CEPAL.

Coy, S., Shipley M., Omer, K., \& Khan, R. (2007). Factors Contributory to Success: a Study of Pakistan's Small Business Owners. Journal of Developmental Entrepreneurship, 12(2), 181-198. https:// doi.org/10.1142/S1084946707000617

Cuevas, H., Parga, N., \& Estrada, S. (2020). Incidencia de la innovación en marketing en el rendimiento empresarial: una aplicación basada en modelamiento con ecuaciones estructurales. Estudios Gerenciales, 35(154), 66-79. https://doi.org/10.18046/j.estger.2020.154.3475

Curmei, V., Ionescu, T., \& Popescu, A. (2011). The Premises Of Strategic Marketing Planning Implementation Within Small and Medium Sized Enterprises. Annals of Faculty of Economics, University of Oradea, 1(1) 730-735. https://bit.ly/2UOeXZ8

De la Cruz, I., Morales, J., \& Carrasco, G. (2006). Construcción de un instrumento de evaluación de capacidades en la empresa: Una propuesta metodológica. X Congreso Anual de la Academia de Ciencias Administrativas, A.C. (ACACIA).

Dini, M., \& Stumpo, G. (Coords.) (2018). Mipymes en América Latina: un frágil desempeño y nuevos desafíos para las políticas de fomento. Santiago: Comisión Económica para América Latina y el Caribe (CEPAL). https://bit.ly/2Y4Q0ek

Domínguez, M., Reséndiz, M., \& Corona, M. (2012). Estrategias de mercadotecnia en empresas manufactureras de la zona metropolitana de la ciudad de puebla 2010. Global Conference On Business y Finance, 7(1), 1150-1158.

Esser, K., Hillebrand, W., Messner, D., \& Meyer-Stamer, J. (1996). Competitividad sistémica: nuevo desafío para las empresas y la política. Revista de la Cepal, 59, 39-52.

Franco, J., Restrepo, J., \& Sánchez, J. (2014). La gestión del mercadeo: Un aporte a la competitividad de las pequeñas empresas del sector servicios en Medellín, Colombia. Pensamiento y gestión, 37, 151-175, http://dx.doi.org/10.14482/pege.37.7025

Gaceta Oficial del Distrito Federal (2013). Programa General para el Desarrollo del Distrito Federal (20132018). México: Gobierno del Distrito Federal.

Gamal, A., Haim, H., \& Abdullahi, G. (2020). Effect of entrepreneurial orientation, market orientation and total quality management on performance Evidence from Saudi SMEs. Benchmarking: An International Journal, 27(4). 1503-153. http://dx.doi.org/10.1108/BIJ-08-2019-0391

García, E., \& Álvarez, J. (1996). Factores de éxito y riesgo en la PYME: Diseño e implantación de un modelo para la mejora de la competitividad. Economía industrial, 310, 149-161.

García, J. (1998). Estudio de los factores que condicionan el éxito o fracaso de las PYMES en Andalucía. I Congreso de Ciencia Regional de Andalucía. Andalucía en el Umbral del Siglo XXI. Jerez, España.

García, R., Maldonado, G., \& Martínez, M. (2014). The Relationship between Market Orientation, Entrepreneurial Orientation, and Innovation: Evidence from Mexican SMEs. Journal of Business and Economics, 5(10), 1930-1940.

http://dx.doi.org/10.15341/jbe(2155-7950)/10.05.2014/020

Gómez, M. (2002). Competitividad de las PyMES: ¿Cómo pueden las pequeñas y medianas empresas de Costa Rica competir en el mercado local e internacional? Economía y Sociedad, 19, 127 -143 , septiembre-diciembre.

Gutiérrez, C., \& Nava, R. (2016). La mercadotecnia digital y las pequeñas y medianas empresas: revisión de la literatura. Enl@ce Revista Venezolana de Información, Tecnología y Conocimiento, 13(1), 45-61.

Huck, J. F., \& McEwen, T. (1991). Competencies needed for small business success: perceptions of jamaican entrepreneurs. Journal of Small Business Management, 29(4), 90-93.

Ibarra, M., González, L., \& Demuner, M. (2017). Competitividad empresarial de las pequeñas y medianas empresas manufactureras de Baja California. Estudios Fronterizos, 18(35), 107-130. http://dx.doi.org/10.21670/ref.2017.35.a06.

INEGI (2010). Censos Económicos 2009. Ciudad de México: INEGI. (2019). Encuesta Nacional de Productividad y Competitividad de la PYME, 2018. Ciudad de México: INEGI. (2020). Censos Económicos, 2019. Ciudad de México: INEGI.

Joensuu-Salo, S., Sorama, K., Viljamaa, A., \& Varamäki, E. (2018). Firm Performance among In- 
ternationalized SMEs: The Interplay of Market Orientation, Marketing Capability and Digitalization. Administrative Sciences (2076-3387), 8(3), 31.

https://doi.org/10.3390/admsci8030031

Kotler, P. (2001). Dirección de mercadotecnia. Análisis, planeación y control, 8va. Edición. Pearson Educación.

Lekmat, L., Selvarajah, C., \& Hewege, Ch. (2018). Relationship between Market Orientation, Entrepreneurial Orientation, and Firm Performance in Thai SMEs: The Mediating Role of Marketing Capabilities. International Journal of Business and Economics, 17(3), 213-237.

Lin, C. (1998). Success Factors of Small- and Medium-Sized Enterprises in Taiwan: An Analysis of Cases. Journal of Small Business Management, 36(4), 43-56.

Longenecker, J., Petty, J., Palich, L., \& Hoy, F. (2012). Administración de pequeñas empresas. Lanzamiento y crecimiento de iniciativas de emprendimiento. Cengage Learning Editores, S.A. de C.V.

Luk, S. K. (1996). Success in Hong Kong: Factors Self-Reported by Successful Small Business Owners. Journal of Small Business Management, 34(3), 68-74.

Martínez, J., \& Álvarez, C. (2006). Mapa de Competitividad para el diagnóstico de PYMES. XI Foro de Investigación. Congreso Internacional de Contaduría Administración e Informática. México, D.F., octubre.

Martínez, M., Santero, R. Sánchez, L., \& Marcos, M. (2009). Factores de competitividad de la pyme española 2008. Fundación EOI, Esc.Organiz.Industrial.

Mehran, R., \& Zeinab, F. (2020) The impact of entrepreneurial marketing on innovative marketing performance in small-and medium-sized companies. Journal of Strategic Marketing, 28(2), 136148. https://doi.org/10.1080/0965254X.2018.1488762

Moferrer, D. (2013). Fundamentos de Marketing. Universidad de Jaume I.

Montoya, A., Montoya, I., \& Castellanos, O. (2010). Situación de la competitividad de la Pyme en Colombia: Elementos actuales y retos. Agronomía Colombiana, 28(1),1-13.

Morales, M., \& Castellano, O. (2007). Estrategias para el fortalecimiento de las Pyme de base tecnológica a partir del enfoque de competitividad sistémica. Innovar. Revista de Ciencias Administrativas y Sociales, 17(29),115-136. http://dx.doi.org/10.15446/innovar

Narváez, M., Fernández, G., \& Henríquez, A. (2013). Competitividad de Empresas Turísticas: Un Análisis desde el Enfoque Sistémico. Rev.fac.cienc.econ., XXI(1), 243-260. https://bit.ly/30USQ7h

OECD (1992). Competitiveness. First report to the president and the Congress. Washington D.C.

O'Dwyer, M., Gilmore, A., \& Carson, D. (2009). Innovative marketing in SMEs. European Journal of Marketing, 43(1/2), 46-61, https://doi.org/10.1108/03090560910923238

Pelham, A.M. (1997). Mediating Influences on the Relationship between Market Orientation and Profitability in Small Industrial Firms. Journal of Marketing Theory and Practice, 5(3), 55-76. https://doi.org/10.1080/10696679.1997.11501771

Pelham, A. M. (2000). Market Orientation and Other Potential Influences on Performance in Small and Medium-Sized Manufacturing Firms. Journal of Small Business Management, 38(1), 48-67. https://doi.org/10.12691/jbms-3-4-2

Peñalosa, M., Calderón, G., \& Benavides, G. (2017). La comercialización en las microempresas de la localidad de Santa Fe en la Ciudad de Bogotá. FACE, 17(1) 19-34. https://doi.org/10.24054/01204211.v1.n1.2017.2575

Peterson, R., \& Crittenden, V. (2020). Exploring customer orientation as a marketing strategy of Mexican-American entrepreneurs. Journal of Business Research, 113 139-148. https://doi.org/10.1016/j.jbusres.2018.12.059.

Petkovska, T., Janevski, Z., Takovska, M., \& Majovski, I. (2018). Application of Marketing Research in Small Enterprises in The Republic of Macedonia. Economic Development, 1-2, 59-72.

Piedrahita, M., \& Paz, R. (2010). Gerencia de relaciones con los clientes en la PYME Colombiana. Revista el hombre y la máquina, 35, 101-110.

Pil, F. K., \& Holweg, M. (2003). Exploring Scale: The Advantages of Thinking Small. MIT Sloan Management Review, 44(2), 33-39. 
Quiroga, D. (2003). Modelo matemático para determinar la competitividad de las Pymes. Cuadernos de Investigación y divulgación. Corporación Universitaria Autónoma de Occidente. Cali, Colombia.

Rodríguez, C., \& Fernández, L. (2006). Manufactura textil en México: Un enfoque sistémico. Revista Venezolana de Gerencia, 11(3)5, 335-351.

Rohvein, C., Paravie, D., \& Urrutia, S. (2012). Metodología de evaluación del nivel de competitividad de las pymes. Revista Ciencias Estratégicas, 21(29), 49-68.

Rojas, M., \& Briceño, M. (2006). La Mercadotecnia en las PYMES manufactureras en el sector residual y tradicional del estado de Trujillo. Visión Gerencial, 6(2) 316-327.

Rubio, A., \& Aragón, A. (2006). Competitividad y recursos estratégicos en la Pyme. Revista de empresa, 17, 32-47, julio-septiembre.

Saavedra. M.L., \& Saavedra, M. E. (2014). Las técnicas de mercadotecnia y su relación con las características de las empresas y empresarios: El caso de las Pequeñas Empresas del estado de Hidalgo, México. Revista de Estudios en Administración Contaduría e Informática, 3(7), 1-31.

Saavedra, M. (2014a). La Pyme como generadora de empleo en México. Revista Clío América, 8(16), 153-172. https://doi.org/10.21676/23897848.1350

Saavedra, M. (Coord.) (2014b). La determinación de la competitividad de las Pyme en el Distrito Federal. FCA, Publishing.

Saavedra, M., \& Milla, S. (2017). La competitividad de la Mipyme en el nivel micro: el caso de Querétaro, México. En-Contexto 5(7), 107-135. https://doi.org/10.15558/fir.v2i4.38

Sánchez, J., Cabanelas, P., Lampón, J., \& González, T. (2019). The impact on competitiveness of customer value creation through relationship capabilities and marketing innovation. Journal of Business $\mathcal{B}$ Industrial Marketing, 34(3), 618-627. https://doi.org/10.1108/JBIM-03-2017-0081

Secretaría de Economía (2009). Criterios de estratificación empresarial. México: Diario Oficial de la Federación, 30 de junio.

Secretaría de Desarrollo Económico (2019). Guía para la inversión 2019 CDMX. Ciudad de México.

Sheetal, Sangeeta \& Kumar, R. (2012). Marketing Strategies of Small and Medium Enterprises: A Sample Survey. International Journal of Management Sciences, 1(2), 60-71. https://bit.ly/3hrzsVk

Schlesinger, M., \& Useche, M. (2005). Mercadeo en las pymes y cooperativas en el estado Zulia. Revista Escuela de Administración de Negocios, 126-135, enero-abril. https://bit.ly/3fuaq5Y

Schnarch, A. (2013). Marketing para PYMES. Un enfoque para Latinoamérica. Alfaomega.

Siu, W.S., Fang, W., \& Lin, T. (2004). Strategic marketing practices and the performance of Chinese small and medium-sized enterprises (SMEs) in Taiwan. Entrepreneurship $\mathcal{E}$ Regional Development, 16(2), 161-178. https://doi.org/10.1080/08985620410001677862

Solleiro, J., \& Castañon, R. (2005). Competitiveness and innovation systems: the challenges for México's. Technovation, 45, 1059-1070. https://doi.org/10.1016/j.technovation.2004.02.005

Mone-Sorina, D., Pop-Marius, D., \& Racolta-Paina N.D. (2013). Marketing Performance in Romanian Small and Medium-Sized Enterprises. A Qualitative Study. Annals of the University of Oradea, Economic Science Series, 22(2), 664-671.

Verhees, F.M., \& Meulenberg, M.G. (2004). Market Orientation, Innovativeness, Product Innovation, and Performance in Small Firms. Journal of Small Business Management, 42(2), 134-154. https://doi.org/10.1111/j.1540-627X.2004.00102.x

Wai-Sum, S. (2005). An institutional analysis of marketing practices of small and medium-sized enterprises (SMEs) in China, Hong Kong and Taiwan. Entrepreneurship y Regional Development, 17, January, 65-88. https://doi.org/10.1080/0898562052000330306

Warren, L., \& Hutchinson, W. E. (2000). Success Factors For High-Technology SMEs: A Case Study From Australia. Journal of Small Business Management, 38(3), 86-91. https://bit.ly/2YR0bDf

Yan, S., \& Chew, D.A.S. (2011). An investigation of marketing strategy, business environment and performance of construction SMEs in China. African Journal of Business Management, 5(6), 23962405. https://doi.org/10.5897/AJBM10.1153

Zapata, E. (2001). La efectividad del mercadeo en las pequeñas y medianas empresas (Pymes) de los sectores industrial y de servicios del departamento de Boyacá, Colombia. Revista Colombiana de Marketing, 2(3), 1-11. https://bit.ly/2ADdBK1 